\title{
The Effectiveness and Value of Rivaroxaban and Icosapent Ethyl as Additive Therapies for Cardiovascular Disease
}

\author{
A Summary from the Institute for Clinical and Economic Review's \\ Midwest Comparative Effectiveness Public Advisory Council \\ Patricia G. Synnott, MS, MALD; R. Brett McQueen, PhD; \\ Daniel A. Ollendorf, PhD; Jonathan D. Campbell, PhD; and Steven D. Pearson, MD, MSc
}

A lthough low-dose aspirin (ASA) and statins have become cornerstone therapies in the management of cardiovascular disease (CVD), this population remains at high residual risk of recurrent cardiovascular events. ${ }^{1}$ The Institute for Clinical and Economic Review (ICER) reviewed the comparative clinical effectiveness and cost-effectiveness of rivaroxaban (Xarelto) and icosapent ethyl (Vascepa) as additive therapies for the management of CVD. Rivaroxaban is an oral direct and selective inhibitor of factor $\mathrm{Xa}$ in the blood coagulation pathway. Icosapent ethyl is a purified ethyl ester of the omega-3 fatty acid known as eicosapentaenoic acid (EPA).

\section{Summary of Findings}

\section{Clinical Effectiveness: Rivaroxaban}

The primary source of evidence for our evaluation of rivaroxaban plus ASA versus ASA alone was the phase 3, randomized COMPASS trial in patients with stable coronary artery disease, peripheral artery disease, or both. ${ }^{2}$ In this trial, rivaroxaban plus ASA reduced the risk of cardiovascular death, stroke, or myocardial infarction (MI) by $24 \%$ (hazard ratio $[\mathrm{HR}]=0.76$; 95\% confidence interval $[\mathrm{CI}]=0.66-0.86)$ but significantly increased the risk of major bleeding $(\mathrm{HR}=1.70 ; 95 \% \mathrm{CI}=1.40$ 2.05). ${ }^{2}$ Major bleeds occurred most commonly in the gastrointestinal tract and led to permanent discontinuation of rivaroxaban plus ASA in $2.7 \%$ of patients. ${ }^{2}$

Based on input from clinical experts, we also sought to compare rivaroxaban plus ASA with dual antiplatelet therapy (DAPT) with ASA and a P2Y12 inhibitor but did not identify any studies that directly compared the 2 regimens. Two trials of ticagrelor plus ASA and clopidogrel plus ASA, respectively, allowed us to conduct a network meta-analysis in the subgroup that experienced an MI within approximately 2 years before randomization. ${ }^{3-6}$ The analysis did not reveal statistical differences between regimens in the comparative risk of cardiovascular death, stroke, or MI. However, given the elevated risk of bleeding that is associated with antithrombotic therapies, any comparative effectiveness analysis is incomplete without an accompanying safety analysis; differences in trial definitions of major bleeding precluded such an analysis.

J Manag Care Spec Pharm. 2020;26(6):782-85

Copyright $\odot 2020$, Academy of Managed Care Pharmacy. All rights reserved.

\section{Clinical Effectiveness: Icosapent Ethyl}

The primary source of evidence for our evaluation of icosapent ethyl was the phase 3, randomized, placebo-controlled REDUCE-IT trial in patients with established CVD or with diabetes and additional risk factors; eligible patients were on a stable dose of statins. ${ }^{7}$ Icosapent ethyl reduced the risk of a composite primary endpoint of cardiovascular death, stroke, MI, coronary revascularization, or unstable angina by $25 \%$ ( $\mathrm{HR}=0.75 ; 95 \% \mathrm{CI}=0.68-0.83)$; the trial's key secondary endpoint, cardiovascular death, stroke, or MI, also occurred in fewer patients treated with icosapent ethyl. ${ }^{7}$ Treatmentemergent adverse events that occurred with greater frequency in the icosapent ethyl group included peripheral edema, constipation, and atrial fibrillation.

Full details from ICER's review are available in the final evidence report at https://icer-review.org/wp-content/uploads/2019/02/ICER_CVD_Final_Evidence_ Report_101719.pdf. ${ }^{8}$

\section{Limitations of the Clinical Evidence}

The COMPASS trial of rivaroxaban recruited patients with a high risk of recurrent cardiovascular events, but low bleeding risk. In addition, clinical benefits observed in the trial may be somewhat overstated due to early termination of the trial for benefit after a mean follow-up of 23 months.

Results of the REDUCE-IT trial of icosapent ethyl stand apart from many previous studies of omega-3 preparations that showed little to no cardiovascular benefit. Several hypotheses have been posited, including the use of a highly purified EPAonly formulation and the use of a higher daily dose (4 grams/day) than previously studied. It remains unclear, however, whether the results of REDUCE-IT would be reproduced in a confirmatory trial and whether the cardioprotective effects of icosapent ethyl are achievable with alternative omega-3 preparations.

Biomarker changes in the REDUCE-IT trial suggest that the mineral oil-containing placebo may not have been biologically inert; patients in the placebo arm experienced increases in low-density lipoprotein and high-sensitivity C-reactive protein. These changes raise the possibility that the true effect of icosapent ethyl may be attenuated from that observed in the trial. In addition, it is uncertain whether icosapent ethyl would be effective in patients who are not on statin therapy. 
TABLE 1 Health Care Sector Cost-Effectiveness Results for Rivaroxaban and Icosapent Ethyl2,7,8

\begin{tabular}{|c|c|c|c|c|c|c|c|}
\hline Intervention $^{\mathrm{a}}$ & $\begin{array}{c}\text { Incremental } \\
\text { Cost, } \$\end{array}$ & $\begin{array}{l}\text { Incremental } \\
\text { Life Years }\end{array}$ & $\begin{array}{c}\text { Incremental } \\
\text { evLYG }\end{array}$ & $\begin{array}{l}\text { Incremental } \\
\text { QALYs }\end{array}$ & $\begin{array}{c}\text { Cost per } \\
\text { Additional } \\
\text { Life Year, \$ }\end{array}$ & $\begin{array}{l}\text { Cost per } \\
\text { Additional } \\
\text { evLYG, } \$\end{array}$ & $\begin{array}{c}\text { Cost per } \\
\text { Additional } \\
\text { QALY, \$ }\end{array}$ \\
\hline Rivaroxaban plus ASA vs. ASA alone & 13,000 & 0.41 & 0.38 & 0.37 & 32,000 & 35,000 & 36,000 \\
\hline $\begin{array}{l}\text { Icosapent ethyl plus optimal medical management } \\
\text { vs. medical management alone }\end{array}$ & 9,000 & 0.54 & 0.52 & 0.50 & 17,000 & 17,000 & 18,000 \\
\hline
\end{tabular}

${ }^{a}$ Modeled populations differed across interventions; results for the interventions are not directly comparable.

$A S A=$ aspirin; evLYG = equal value life-years gained evenly measures any gains in length of life, regardless of the impact on patients' quality of life; QALY=quality-adjusted life-year.

\section{Long-Term Cost-Effectiveness}

We developed a Markov model to compare the cost-effectiveness of rivaroxaban plus ASA with ASA alone and to compare the addition of icosapent ethyl to optimal medical management (including statins) to optimal medical management alone. Both therapies were modeled separately using the same model structure but with distinct populations and model inputs. The model estimated patient survival, quality-adjusted survival, and health care costs over a lifetime time horizon for each intervention and comparator. The base-case analysis used a U.S. health care system perspective with a 3\% annual discount rate for costs and health outcomes.

Modeled populations began treatment and could stay in that state or pass into event states of MI, stroke, or death. Those who experienced a cardiovascular event moved into postevent health states, where they could remain or transition to another cardiovascular event state. The model tracked all patients until death. All patients could transition to death from any of the health states, and death could result from all-cause or cardiovascular-related causes.

Results from our model showed that at the estimated annual net prices of $\$ 2,215$ and $\$ 1,625$ for rivaroxaban and icosapent ethyl, respectively, incremental cost-effectiveness ratios were $\$ 36,000$ per quality-adjusted life-year (QALY) for rivaroxaban in comparison with ASA alone and $\$ 18,000$ per QALY for icosapent ethyl versus medical management alone; additional results (incremental cost per life-year and per equal value lifeyears gained) are presented in Table $1 .{ }^{9}$ The results were relatively robust to sensitivity and scenario analyses. In probabilistic sensitivity analyses, $100 \%$ of model iterations resulted in an additional cost-per-QALY result below a threshold of $\$ 100,000$ per QALY gained for both therapies.

ICER's potential budget impact analysis suggested that only approximately $5 \%$ of eligible patients with CVD could be treated with either drug before passing a budget impact threshold of $\$ 819$ million per year over 5 years. Full details on ICER's analyses of cost-effectiveness and potential budget impact, including sensitivity and scenario analyses, are available in the final evidence report on the ICER website. ${ }^{8}$

\section{Limitations of the Cost-Effectiveness Model}

The results of our analysis were relatively robust to sensitivity and scenario analyses. However, given that the model relied on randomized controlled trial estimates of clinical benefit and harm, the findings should be interpreted with caution when estimating whether these interventions would achieve similar value for money in actual practice.

\section{Policy Discussion}

The Midwest Comparative Effectiveness Public Advisory Council (CEPAC; https://icer-review.org/programs/midwestcepac/) is an independent appraisal committee convened by ICER to engage in the public deliberation of the evidence on clinical effectiveness and cost-effectiveness of health care interventions. CEPAC is composed of medical evidence experts and leaders in patient advocacy. Their deliberation includes input from clinical experts and patient representatives specific to the condition under review, as well as formal comment from the public. A policy roundtable concludes each meeting during which representatives from insurers and manufacturers join with clinical experts and patient representatives to discuss how best to apply the findings of the evidence to clinical practice, insurance coverage, and pricing negotiations.

The ICER report on additive therapies for CVD was the subject of a CEPAC meeting in September 2019. Following discussion, the CEPAC panel voted 10-1 that the evidence was adequate to demonstrate that the net health benefit provided by rivaroxaban plus ASA is superior to that provided by ASA alone. For the comparison of rivaroxaban plus ASA to DAPT, the panel unanimously voted that there was not adequate evidence to demonstrate that rivaroxaban provided a superior net health benefit. The panel voted 9-2 that the net health benefit provided by icosapent ethyl plus optimal medical management (including statins) is superior to that provided by optimal medical management alone.

The CEPAC panel also voted on "potential other benefits" and "contextual considerations" of both therapies as part of a process intended to signal to policymakers whether there are important considerations when making judgments about long-term value for money that are not adequately captured 


\section{TABLE 2 Potential Other Benefits, Disadvantages, and Contextual Considerations}

\begin{tabular}{|c|c|c|c|c|c|}
\hline $\begin{array}{l}\text { Does treating patients with } \\
\text { the intervention offer } 1 \text { or } \\
\text { more of the following } \\
\text { potential "other benefits or } \\
\text { disadvantages"? }\end{array}$ & $\begin{array}{l}\text { Rivaroxaban plus } \\
\text { ASA vs. ASA alone }\end{array}$ & $\begin{array}{l}\text { Icosapent ethyl } \\
\text { plus optimal } \\
\text { medical } \\
\text { management } \\
\text { (including statin } \\
\text { therapy) vs. } \\
\text { optimal medical } \\
\text { management } \\
\text { alone }\end{array}$ & $\begin{array}{l}\text { Are any of the following contex- } \\
\text { tual considerations important in } \\
\text { assessing the long-term value for } \\
\text { money of the interventions? }\end{array}$ & $\begin{array}{l}\text { Rivaroxaban plus } \\
\text { ASA vs. ASA alone }\end{array}$ & $\begin{array}{l}\text { Icosapent ethyl } \\
\text { plus optimal } \\
\text { medical } \\
\text { management } \\
\text { (including statin } \\
\text { therapy) vs. } \\
\text { optimal medical } \\
\text { management alone }\end{array}$ \\
\hline $\begin{array}{l}\text { This intervention will sig- } \\
\text { nificantly reduce caregiver or } \\
\text { broader family burden }\end{array}$ & $1 / 11$ & $0 / 11$ & $\begin{array}{l}\text { This intervention is intended for } \\
\text { the care of individuals with a con- } \\
\text { dition of particularly high severity } \\
\text { in terms of impact on length of life } \\
\text { and/or quality of life }\end{array}$ & $6 / 11$ & $6 / 11$ \\
\hline $\begin{array}{l}\text { This intervention offers a } \\
\text { novel mechanism of action } \\
\text { or approach that will allow } \\
\text { successful treatment of many } \\
\text { patients for whom other avail- } \\
\text { able treatments have failed }\end{array}$ & $6 / 11$ & $7 / 11$ & $\begin{array}{l}\text { This intervention is intended for } \\
\text { the care of individuals with a con- } \\
\text { dition that represents a particu- } \\
\text { larly high lifetime burden of illness }\end{array}$ & $6 / 11$ & $6 / 11$ \\
\hline $\begin{array}{l}\text { This intervention will have a } \\
\text { significant impact on improv- } \\
\text { ing patients' ability to return } \\
\text { to work and/or their overall } \\
\text { productivity }\end{array}$ & $1 / 11$ & $0 / 11$ & $\begin{array}{l}\text { There is significant uncertainty } \\
\text { about the long-term risk of serious } \\
\text { side effects of this intervention }\end{array}$ & 9/11 & $5 / 11$ \\
\hline \multirow[t]{2}{*}{$\begin{array}{l}\text { There are other important } \\
\text { benefits or disadvantages that } \\
\text { should have an important role } \\
\text { in judgments of the value of this } \\
\text { intervention. }\end{array}$} & No vote & No vote & $\begin{array}{l}\text { There is significant uncertainty } \\
\text { about the magnitude or durability } \\
\text { of the long-term benefits of this } \\
\text { intervention }\end{array}$ & $9 / 11$ & $7 / 11$ \\
\hline & & & $\begin{array}{l}\text { There are additional contextual } \\
\text { considerations that should have an } \\
\text { important role in judgments of the } \\
\text { value of this intervention }\end{array}$ & No vote & No vote \\
\hline
\end{tabular}

$A S A=$ aspirin

in the analyses of clinical effectiveness and cost-effectiveness. The results of these votes are shown in Table 2 and highlight several factors that the CEPAC panel felt were important for judgments of value.

The culminating vote of the CEPAC panel, intended to integrate the elements of the value assessment framework, is normally on the "long-term value for money." As described in ICER's 2017-2019 value assessment framework, questions on long-term value for money are subject to a value vote when incremental cost-effectiveness ratios for the interventions of interest are between $\$ 50,000$ and $\$ 175,000$ per QALY in the "base case" analysis. The base case estimates of the cost per QALY for rivaroxaban and icosapent ethyl fell below the lower end of this range; therefore, they were deemed "high long-term value for money" without a vote. There was no value vote taken for the comparison of rivaroxaban plus ASA to DAPT because the evidence was considered inadequate during the clinical vote.

The policy roundtable discussion explored how best to translate the evidence and additional considerations into clinical practice and into pricing and insurance coverage policies.
The full set of recommendations can be found in the final evidence report. Key policy recommendations are as follows:

- DAPT should not be considered in a step therapy protocol as a first step before receiving coverage for rivaroxaban.

- A decision tool should be developed to help determine appropriate additive therapies for an individual patient.

- The U.S. Food and Drug Administration, manufacturers, and the clinical research community should standardize key outcome definitions such as major bleeding.

As noted, ICER's potential budget impact analysis suggested that only approximately $5 \%$ of eligible patients with CVD could be treated with either drug before passing a budget impact threshold of $\$ 819$ million per year over 5 years. During the policy discussion, clinical experts stated that they would consider using rivaroxaban in approximately $30 \%$ of eligible patients and icosapent ethyl in the majority of eligible patients. ICER therefore issued an "Access and Affordability Alert" in its final report to signal to policymakers that special measures may need to be considered to ensure that the health care system can absorb the added costs from these therapies over the short 
term without reducing access inappropriately or contributing to rapid growth in health care insurance costs.

\section{Conclusions}

Tremendous health gains have been achieved with inexpensive treatments such as aspirin and statins, but high-risk patients continue to be at substantial risk of cardiovascular events even on these treatments. ICER's evidence review suggests that rivaroxaban and icosapent ethyl can provide additional benefits at costs that fall within commonly cited cost-effectiveness thresholds. Nevertheless, the eligible population sizes for both therapies are large, and sustainable access to high-value care for all patients will require thoughtful approaches to managing short-term access and affordability.

\section{Authors}

PATRICIA G. SYNNOTT, MS, MALD, and STEVEN D. PEARSON, MD, MSc, Institute for Clinical and Economic Review, Boston, Massachusetts. R. BRETT MCQUEEN, PhD, and JONATHAN D. CAMPBELL, PhD, Skaggs School of Pharmacy and Pharmaceutical Sciences, University of Colorado Anschutz Medical Campus, Aurora. DANIEL A. OLLENDORF, PhD, Center for the Evaluation of Value and Risk in Health, Tufts Medical Center, Boston, Massachusetts.

AUTHOR CORRESPONDENCE: Patricia G. Synnott, MS, MALD, Center for the Evaluation of Value and Risk in Health, Institute for Clinical Research and Health Policy Studies, Tufts Medical Center, 800 Washington St., Box 63, Boston, MA 02111

E-mail: psynnott@tuftsmedicalcenter.org.

\section{DISCLOSURES}

Funding for this summary was contributed by Arnold Ventures, Commonwealth Fund, California Health Care Foundation, National Institute for Health Care Management (NIHCM), New England States Consortium Systems Organization, Blue Cross Blue Shield of Massachusetts, Harvard Pilgrim Health Care, Kaiser Foundation Health Plan, and Partners HealthCare to the Institute for Clinical and Economic Review (ICER), an independent organization that evaluates the evidence on the value of health care interventions.
ICER's annual policy summit is supported by dues from Aetna, America's Health Insurance Plans, Anthem, Allergan, Alnylam, AstraZeneca, Biogen, Blue Shield of CA, Cambia Health Services, CVS, Editas, Express Scripts, Genentech/Roche, GlaxoSmithKline, Harvard Pilgrim, Health Care Service Corporation, Health Partners, Johnson \& Johnson (Janssen), Kaiser Permanente, LEO Pharma, Mallinckrodt, Merck, Novartis, National Pharmaceutical Council, Premera, Prime Therapeutics, Regeneron, Sanofi, Spark Therapeutics, and United Healthcare.

Pearson is employed by ICER; Synnott was employed by ICER at the time of this report. Ollendorf, Campbell, and McQueen received grants from ICER for work on this review. Ollendorf also reports advisory board, consulting, and other fees from Sarepta Therapeutics, DBV Technologies, EMD Serono, Gerson Lehman Group, The CEA Registry Sponsors, Autolus, Analysis Group, Amgen, AbbVie, Cytokinetics, Aspen Institute/University of Southern California, and University of Colorado, unrelated to this review.

\section{ACKNOWLEDGMENTS}

The authors thank David M. Rind, MD, MSc; Ellie Adair, MPA; Serina HerronSmith; and Katherine Fazioli for their contributions to ICER's Midwest CEPAC report on additive therapies for cardiovascular disease.

\section{REFERENCES}

1. Patel R, Tragante V, Schmidt AF, et al. Subsequent event risk in individuals with established coronary heart disease: design and rationale of the GENIUS-CHD Consortium. Circ Genom Precis Med. 2019;12(4):e002470.

2. Eikelboom JW, Connolly SJ, Bosch J, et al. Rivaroxaban with or without aspirin in stable cardiovascular disease. N Engl J Med. 2017;377(14):1319-30.

3. Bonaca MP, Bhatt DL, Cohen M, et al. Long-term use of ticagrelor in patients with prior myocardial infarction. N Engl J Med. 2015;372(19):1791-800.

4. Bonaca MP, Storey RF, Theroux P, et al. Efficacy and safety of ticagrelor over time in patients with prior MI in PEGASUS-TIMI 54. J Am Coll Cardiol. 2017;70(11):1368-75.

5. Bhatt DL, Fox KAA, Hacke W, et al. Clopidogrel and aspirin versus aspirin alone for the prevention of atherothrombotic events. N Engl J Med. 2006;354(16):1706-17.

6. Bhatt DL, Flather MD, Hacke W, et al. Patients with prior myocardial infarction, stroke, or symptomatic peripheral arterial disease in the CHARISMA Trial. J Am Coll Cardiol. 2007;49(19):1982-88.

7. Bhatt DL, Steg PG, Miller M, et al. Cardiovascular risk reduction with icosapent ethyl for hypertriglyceridemia. N Engl J Med. 2019;380(1):11-22.

8. Ollendorf D, McQueen R, Campbell J, et al. Additive therapies for cardiovascular disease: effectiveness and value. Institute for Clinical and Economic Review. October 17, 2019. Available at: http://icer-review.org/material/cvdfinal-evidence-report/. Accessed April 30, 2020.

9. SSRHealth. U.S. Brand Rx Net Price. 2019. Available at: https://www. ssrhealth.com/dataset/. Accessed April 30, 2020. 